TITRE: RECRUTEMENT EN PHASE 1 CHEZ DES PARTICIPANTS ATTEINTS D'UNE MALADIE GRAVE

Auteur(s): François POULIOT

PublicAtION: RECRUTEMENT ET CONSENTEMENT À LA RECHERCHE : RÉALITÉS ET DÉFIS ÉTHIQUES

PAGES: $65-71$

ISBN: 978-2-7622-0357-8

Directeurs: Ana Marin, BéATrice Eysermann et Michel T. GIROUX

URI: HTTP://HDL.HANDLE.NET/11143/14109

DOI: HTTPS://DOI.ORG/10.17118/11143/14109 


\section{Recrutement en phase 1 chez des participants atteints d'une maladie grave}

François Pouliot, M.D., Ph. D., vice-président du Comité d'éthique de la recherche du CHU de Québec-Université Laval

Résumé : Si les noms TeGenero et Bial sont associés à des tragédies chez des volontaires sains, ils ne doivent pas faire oublier les problèmes plus fréquents et tout aussi sérieux chez les participants atteints d'une maladie grave comme le cancer. La différence fondamentale est qu'il n'existe pour les volontaires sains aucun intérêt thérapeutique à participer à une telle recherche.

La première utilisation de nouvelles molécules chez l'humain, appelée couramment phase 1, cherche à étudier la sécurité du produit et à établir la dose optimale. En effet, l'identification de cibles moléculaires précises, la fabrication de molécules sur mesure, le phénomène de résistance, l'urgence d'offrir une certaine alternative à des patients en fin de course incitent à utiliser le nouveau produit plus rapidement et auprès d'une plus large population. Si l'on a pu accéder rapidement aux nouveaux médicaments contre le SIDA, on devrait faire de même contre le cancer.

Au plan éthique, plusieurs voient un intérêt thérapeutique pour tout malade participant à la recherche, même lors de la toute première utilisation. Si les bénéfices sont habituellement peu importants, le rapport risques/ bénéfices est souvent intéressant. D'ailleurs, la motivation première de ces participants est le plus souvent l'amélioration de leur situation, non la production de connaissances. Plus radicalement encore, des patients sont prêts à prendre de grands risques pour la recherche sur leur maladie ou d'autres problèmes. Au regard de leur autonomie et de leur capacité à consentir, la prudence des autorités concernées apparaît à certains patients comme une forme dépassée de paternalisme.

Mots-clés : recherche, phase 1, participants, autonomie, paternalisme 


\section{Introduction}

En 2006, une jeune compagnie nommée TeGenero fit la manchette des médias parce que six participants à la recherche se retrouvèrent aux soins intensifs à la suite de l'utilisation d'une substance modifiant les réactions immunitaires du corps humain. L'ensemble des volontaires avaient reçu leur dose de médicament à peu près en même temps, ce qui avait empêché les chercheurs de prévenir les mêmes effets secondaires chez les participants ${ }^{1}$. En 2015, la compagnie Bial reçut le même genre de publicité quand plusieurs participants en bonne santé présentèrent des symptômes graves après une première dose d'un nouveau produit². Ces deux tragédies ne doivent pas faire oublier des problèmes plus fréquents et tout aussi sérieux qui se retrouvent chez des participants atteints de maladies graves comme le cancer ou la sclérose en plaques. La différence fondamentale entre ces deux groupes est que les volontaires sains n'ont aucun intérêt thérapeutique à participer à une telle recherche.

La toute première utilisation de nouvelles molécules chez des patients gravement malades est particulièrement présente en oncologie. Au MD Anderson Cancer Center, par exemple, environ 800 personnes atteintes de cancer parmi les 11000 participants à la recherche sont inscrites dans des projets de phase 1 répartis en 110 projets $^{3}$. Ces projets sont souvent des thérapies moléculaires ciblées (Molecular Targeted Therapies) visant des récepteurs cellulaires spécifiques.

La question est d'une grande actualité et représente un problème éthique significatif. N'est-ce pas exploiter des personnes grandement vulnérables, prêtes à accepter tout projet de recherche, même risqué, au nom de la science alors que le désir de vivre les pousse aussi à le faire? Des professionnels de la santé et des chercheurs consciencieux peuvent-ils laisser à des personnes non expertes l'évaluation des risques et des bénéfices d'un tel projet de recherche? De quel droit veut-on imposer à ces personnes un certain niveau de risque acceptable alors qu'elles sont prêtes à prendre plus de risques?

\section{Le développement d'une nouvelle molécule}

Le développement d'une nouvelle molécule est un long et complexe processus. Après les recherches fondamentales en laboratoire puis sur les animaux arrive le moment où le produit montre suffisamment d'intérêt pour être testé chez l'humain pour la première fois. Cette étape, appelée phase 1, cherche à étudier la sécurité du produit, à établir la dose optimale (quantité, fréquence et voie d'administration) et à documenter les premiers signes d'efficacité. Une telle étude compte entre 15 et 30 participants, regroupés en petites cohortes de trois à cinq personnes.

Quand les résultats de cette première étape le permettent, la phase 2 est enclenchée. Celle-ci continue à récolter les données de sécurité, mais vise à vérifier si le nouveau médicament a une certaine efficacité quant au problème étudié. Elle compte souvent moins de 100 participants.

La phase 3 veut comparer l'efficacité du nouveau produit avec celle du traitement considéré comme standard. Des centaines, voire des milliers de participants sont recrutés pour une telle recherche. Quand tout se passe

1. Voir http://www.nytimes.com/2006/04/07/world/europe/when-drug-trials-go-horribly-wrong.html

2. Le journal Le Figaro a publié une série d’articles sur le groupe Bial. Voir par exemple : Essai thérapeutique. Bial assure avoir respecté "les bonnes pratiques», dans l'édition du 15 janvier 2016, ou encore Rennes. L'agence du médicament publie le protocole de Bial, dans celle du 22 janvier 2016.

3. Cf. Messenger, MD Anderson Cancer Center, Houston, July/August 2010, p. 5. 
bien, cette étape aboutit à une approbation des autorités en la matière, notamment Santé Canada, et à la commercialisation du produit.

Enfin, la phase 4 survient après cette commercialisation. Elle cherche à examiner de nouvelles indications possibles. Des informations sur la sécurité acquises auprès des utilisateurs du produit sont collectées.

\section{Quelques enjeux éthiques liés à la participation des personnes atteintes d'une maladie grave aux recherches de phase 1}

\section{Le recrutement de participants malades}

La règle générale veut que la recherche de phase 1 se fasse avec des volontaires sains. Parfois, celle-ci n'est pas possible à cause de la spécificité du produit à l'étude (par exemple, un anticorps dirigé contre des protéines anormales retrouvées dans les cellules cancéreuses) ou encore de ses graves dangers contre l'organisme (effets de la thalidomide et de ses dérivés sur le développement intra-utérin). II n'en reste pas moins que, dans la majorité des cas, la sécurité du produit n'est pas établie tant qu'il n'est pas utilisé chez l’humain.

Le recrutement de participants malades est donc souvent nécessaire, mais il fait l'objet de restrictions importantes. Dans la Déclaration d'Helsinki de l'Association médicale mondiale (AMM, 2013) sur les principes éthiques applicables à la recherche médicale impliquant des êtres humains, la priorité a été accordée à la protection des participants. La Déclaration en fait un devoir du médecin :

4. Le devoir du médecin est de promouvoir et de sauvegarder la santé, le bien-être et les droits des patients, y compris ceux des personnes impliquées dans la recherche médicale. Le médecin consacre son savoir et sa conscience à l'accomplissement de ce devoir [...].

9. Il est du devoir des médecins engagés dans la recherche médicale de protéger la vie, la santé, la dignité, l'intégrité, le droit à l'autodétermination, la vie privée et la confidentialité des informations des personnes impliquées dans la recherche. La responsabilité de protéger les personnes impliquées dans la recherche doit toujours incomber à un médecin ou à un autre professionnel de santé et jamais aux personnes impliquées dans la recherche même si celles-ci ont donné leur consentement.

On a ainsi voulu préserver une population vulnérable et non experte contre les investigateurs et contre ellemême. Selon la Déclaration d'Helsinki (AMM, 2013), le simple fait d'appartenir à un groupe vulnérable, que ce soit au plan physique, psychologique, social ou économique, implique que la recherche doit répondre aux besoins ou aux priorités sanitaires de ce groupe. Ce groupe devrait aussi bénéficier des connaissances, des pratiques ou interventions qui en résultent (AMM, 2013, art 20). Le médecin peut aussi recourir à une intervention non avérée si elle offre une chance de sauver la vie, rétablir la santé ou alléger les souffrances du patient quand il n'y a pas d'intervention avérée («traitement standard») ou faute d'efficacité de ces interventions (AMM, 2013, art. 37).

Or, de plus en plus, les participants eux-mêmes veulent fixer le niveau de risque acceptable par rapport aux bénéfices attendus en fonction de leurs objectifs et valeurs. Aux États-Unis, l'influence du groupe de pression ACT UP sur le développement de la trithérapie contre le SIDA est un bon exemple de l'impact de patients devenus activistes. Alors que les organismes de régulation des médicaments voulaient s'assurer de la sécurité maximale des nouvelles substances, ces groupes insistaient pour avoir accès rapidement à celles-ci dès qu'elles montraient un bénéfice significatif. Des regroupements de patients ont aussi fait pression sur la FDA 
afin d'accélérer l'accès à l'Irinotecan, un médicament prometteur contre le cancer du côlon (Daugherty, 1997; Wachter, 1992; Rothenberg, M.L, Eckart, J.R., Kahn, J.G. et coll., 1996). Ces exemples montrent que certains participants contestent une certaine forme de paternalisme voulant les protéger contre eux-mêmes et réclament de pouvoir choisir eux-mêmes le niveau de risque jugé acceptable. Un tel conflit entre obligation professionnelle et autodétermination ne trouvera de solution éthiquement acceptable qui si les deux groupes évoluent vers un partenariat qui tiendra compte des risques et des bénéfices établis par la science et qui veillera méticuleusement à respecter le processus de consentement.

\section{Le rapport entre les risques et les bénéfices}

La volonté de participer à un essai clinique ne peut suffire pour y avoir accès. Il doit exister une forme de proportion ou d'équilibre qui justifie l'utilisation d'un tel traitement. La Déclaration indique, à l'article 16, qu'une «recherche médicale impliquant des êtres humains ne peut être conduite que si l'importance de l'objectif dépasse les risques et inconvénients pour les personnes impliquées». Le Code civil du Québec affirme, à l'article 20, qu'une «personne majeure, apte à consentir, peut participer à une recherche susceptible de porter atteinte à son intégrité pourvu que le risque couru ne soit pas hors de proportion avec le bienfait qu'on peut raisonnablement en espérer».

Le Code civil est cependant plus restrictif pour les mineurs et les majeurs inaptes, la recherche devant laisser espérer un bienfait ou des résultats bénéfiques4

Les études de phase 1 peuvent avoir un impact positif sur la santé des participants. Les données quantitatives compilées par Agrawal et Emanuel (Agrawal et Emanuel, 2008) montrent des résultats globaux modestes dans le domaine de l'oncologie : on parle de réponse dans 4,4 \% des cas : une réponse complète dans 1,9\% des cas, une réponse partielle (correspondant à au moins $50 \%$ de diminution de la tumeur) dans 2,5\% des cas. Cependant, certaines réponses spectaculaires ont été notées : le cancer médullaire de la thyroïde avec le XL184; le cancer de l'ovaire avec le patupilone; le sarcome d'Ewing avec des inhibiteurs de l'IGFR; la maladie de Castleman avec l'anti-interleukine-6; le cancer des testicules avec le cisplatine (une réponse a été notée dans $50 \%$ des cas, une guérison dans $25 \%$ ); la leucémie myéloïde chronique avec le Gleevec (une réponse hématologique a été notée dans $98 \%$ des cas).

Du côté des risques, la même source parle d'une mortalité à 0,5\% et d'une toxicité importante (niveaux 3 et 4) chez $10 \%$ des participants à des études de phase 1 en oncologie.

Ces chiffres ne prennent évidemment pas en compte les aspects qualitatifs de la survie liés à la qualité bonne ou mauvaise de la vie, aux bienfaits psychologiques et sociaux tirés de la participation à un projet de recherche, au sentiment de mieux contrôler l'évolution de la maladie, de contribuer à l'évolution de la science et à l'amélioration des soins à venir, pour ne nommer que quelques éléments. Il en va souvent du sens donné à ce qui leur reste de vie (Jansen, Appelbaum, Klein et coll., 2011; Daugherty, Ratain, Grochowski et coll. 1995).

\footnotetext{
4. «Un mineur ou un majeur inapte ne peut participer à une recherche susceptible de porter atteinte à son intégrité qu'à la condition que le risque couru, en tenant compte de son état de santé et de sa condition personnelle, ne soit pas hors de proportion avec le bienfait qu'on peut raisonnablement en espérer. Il ne peut, en outre, participer à une telle recherche qu'à la condition que la recherche laisse espérer, si elle ne vise que lui, un bienfait pour sa santé ou, si elle vise un groupe, des résultats qui seraient bénéfiques aux personnes possédant les mêmes caractéristiques d’âge, de maladie ou de handicap que les membres du groupe.» Code civil du Québec, art. 20.
} 


\section{Le consentement libre et éclairé}

L'étude déjà ancienne de Daugherty et coll. (1995) montrait que les participants en phase 1 sont motivés par la perspective thérapeutique, peu par l'avancement de la science et l'altruisme. Ils ont une perception correcte des risques et des bénéfices, mais méconnaissent le rôle des études de phase 1 dans l'établissement de la dose adéquate. Il y a donc raison d'être prudent quand on propose une étude de phase 1 à des gens vulnérables. Chaque étape doit être réalisée avec grande attention.

Dans le cas où ces patients sont traités dans des unités de soins intensifs ou reçoivent des soins palliatifs, les mesures de précaution seront encore plus importantes. Leur état physique et mental, de même que les conditions très concrètes dans lesquels ils sont placés peuvent interférer avec la possibilité concrète de la recherche. Il est difficile de recevoir l'information pertinente, de réfléchir de façon sereine selon ses valeurs, de communiquer sa décision, sans parler de la réalisation sécuritaire de l'étude elle-même, quand la chambre est pleine d'appareils, que le va-et-vient du personnel dérange ou, au contraire, quand les lieux n'accueillent aucune technologie! Aussi surprenant que cela puisse paraître de prime abord, ils ne sont pas automatiquement exclus d'une participation à une étude de phase 1.

\section{Conclusion}

Dans l'état actuel, il est possible pour des patients atteints d'une maladie grave, mais dans un état fonctionnel relativement bon, de participer à des études de phase 1. Parfois, ce sont les seuls qui peuvent être recrutés. Certains veulent aller loin et sont prêts à prendre des risques importants en participant à ces études de phase 1 . Les avantages et les risques leur ont été expliqués et ils ont été jugés aptes à consentir. Au regard de leur autonomie et de leur capacité à consentir, le refus des autorités concernées à les recruter apparaît à certains comme une forme dépassée de paternalisme. Devrait-on refuser une telle participation uniquement quand l'objet de l'étude n'est pas en lien avec le problème de santé dont ils souffrent? Ne devrait-on pas aussi retenir ceux qui «offrent leurs corps à la science», prêts à recevoir tout ce qu'on leur proposera? Tel semble bien être l'état des lieux en ce moment. 


\section{Bibliographie}

Agrawal, M. et Emanuel, E.J. (2008). Phase 1 Oncology Research. Dans Emanuel, E.J. et coll., The Oxford Textbook of Clinical Research Ethics (p. 356-366). Oxford University Press.

Anderson, J.A. et Kimmelman, J. (2010). Extending Clinical Equipoise to Phase 1 Trials Involving patients : Unresolved Problems, Kennedy Institute of Ethics Journal, 20 (1), 75-98.

Association médicale mondiale (AMM). (2013). Déclaration d'Helsinki de l'AMM-Principes éthiques applicables à la recherche médicale impliquant des êtres humains, Fortaleza.

Code civil du Québec, RLRQ, c. C.c.Q.-1991.

Daugherty, C., Ratain, M.J., Grochowski, E. et coll. (1995). Perceptions of Cancer Patients and Their Physicians involved in Phase I Trials. J Clin Oncol., 13, 1062-1072.

Daugherty, C, Siegler M, Ratain, M.J. et Zimmer, G (1997). Learning from Our Patients: One Participant's Impact on Clinical Trial Research and Informed Consent, Annals of Internal Medicine, 125, 892-897.

Jansen, L.A., Appelbaum, P.S., Klein, W.M.P. et coll. (2011). Unrealistic Optimism in Early-Phase Oncology Trials, IRB: Ethics and Human Research, 33, 1-8.

Rothenberg, M.L., Eckart, J.R., Kahn, J.G. et coll. (1996). Phase II trial of irinotecan in patients with progressive or rapidly recurrent colorectal cancer, J Clin Oncol.,14, 1128-35.

Wachter, R.M. (1992). AIDS, activism, and the politics of health, N Engl J Med., 326, 128-33. 